\title{
MODELLING OF SEDIMENTATION FOR POLYDISPERSE MIXTURE
}

\author{
EWELINA ŁUKASIEWICZ \& MARIUSZ RZĄSA \\ Department of Thermal Engineering and Industrial Facilities, Opole University of Technology, Poland
}

\begin{abstract}
The present study concerns the mathematical description for particle fractions during the sedimentation process. The authors have taken into account polydisperse suspension, which is formed during the coagulation process. In water and wastewater treatment, coagulation is a technological stage for chemical treatment. According to this, alum or other chemicals are added to water to form tiny, sticky particles called flocs. The way that the flocs settle is very difficult to describe. Group classification of the flocs may help to define the sedimentation process for post-coagulation suspensions, and may ensure new mathematical equations for this problem.
\end{abstract}

Keywords: sedimentation, polydisperse suspension, coagulation.

\section{INTRODUCTION}

Mathematical models for the sedimentation of polydisperse suspensions, consisting of small spherical particles belonging to a finite number of species that differ in size or density, are important to a variety of applications such as solid-liquid separation in mineral processing and wastewater treatment, classification fluidization, cast formation in the ceramic industry, blood sedimentation in medicine, and volcanology. Polydisperse suspensions consist of particles differing in size or density that are dispersed in a viscous fluid. During sedimentation, the different particle species segregate and create areas of different composition [1], [2].

One of the processes where polydisperse suspension may be created, is the coagulation process. Coagulation is very important step in industrial and service water and wastewater treatment. The demand for high-quality water is increasing, because the number of uncontaminated water sources decreases. The criteria for the discharge of wastewater are becoming stricter to prevent the environment contamination and/or the infection of drinking water sources. Before use the water must be treated, and must be ensure that it is free of pathogens and chemicals posing a risk to health [3]. Coagulation/flocculation is considered the most important process in surface water treatment. The type of coagulant used has a significant impact on coagulation processes. Aluminium-based coagulants have been more widely used than ferric-based coagulants, probably because aluminium coagulants have a superior NOM (Natural Organic Matter) removal capacity, whereas ferric coagulants create water colour problems [4]-[6]. However, a large amount of alum salts has also been suspected to be harmful to people and other living organisms [7]. Flocs formed in coagulation and flocculation processes settle in a very difficult way. A porous structure and various shapes of created flocs, have an effect on the settling way and velocity. These are the reasons why theoretical description of the sedimentation of floc suspension is only an approximate way of describing this phenomenon.

Coagulation may be carried out as a volume, contact and surface process. Usually, is achieved in a mixing chamber or accelerator with fast mixing time about 1-3 minutes. The second phase of coagulation is flocculation, which involves the occurrence of the phenomena leading to the formation of agglomerates and hydrolysis products of the coagulant dissociated by removing impurities. The volume coagulation is carried out in slow-stirred chambers 
(flocculation), but the contact coagulation is carried out in a layer of solid matter [8]. After mixing chamber water goes to settler where sedimentation process occurs. Flocs can be efficiently removed by the process of sedimentation/flotation and filtration. After mixing process water is directed to clarifiers.

Settling tanks are devices in which there is a process removing the suspension from purified water. They are used to easily retain the falling suspension, natural or produced by the coagulation process and chemical precipitation of sparingly soluble compounds. Contact settlers (Fig. 1) with suspended sediment are used to treatment the water by coagulation and are usually called clarifiers. In settlers of horizontal flow of solid particles, float of particles depends on the flow rate $\left(\mathrm{v}_{\mathrm{p}}\right)$ of water in the sedimentation tank, and descent rate $\left(\mathrm{v}_{0}\right)$. As a result of these two forces, the particles falling down direction is a resultant function between $\mathrm{v}_{\mathrm{p}}$ and $\mathrm{v}_{0}$. The movement of solid particles in a perfect horizontal settler shown in eqn (1). If the settling velocity of particles in the way $\mathrm{H}$ is denoted in eqn (1):

$$
\mathrm{v}_{0}=\frac{\mathrm{H}}{\mathrm{t}_{\mathrm{p}}}
$$

and to calculate flow time through settling tank $\left(t_{p}\right)$ is volume of tank $(V)$ quotient and the flow rate $(\mathrm{Q})$, we have:

$$
\mathrm{v}_{0}=\frac{\mathrm{Q}}{\mathrm{F}}
$$

The particles which will falling with velocity $\mathrm{v} \geq \mathrm{v}_{0}$ will be stopped in settling tank. According to this, the particles with velocity $\mathrm{v}<\mathrm{v}_{0}$ will be stopped only these particles, which are located at the distance $\mathrm{h}=\mathrm{v} \cdot \mathrm{t}_{\mathrm{p}}$ from the bottom of the settling tank (where $0<$ $\mathrm{h}<H)[9]$.

\section{POLYDISPERSE MIXTURE}

Safe drinking water is essential to the health and welfare of a community, and water from all sources must have some form of purification before consumption. A very important step in the process of purifying is a coagulation and flocculation process, which is widely used due to its simplicity and cost-effectiveness. Regardless of the nature of the treated sample

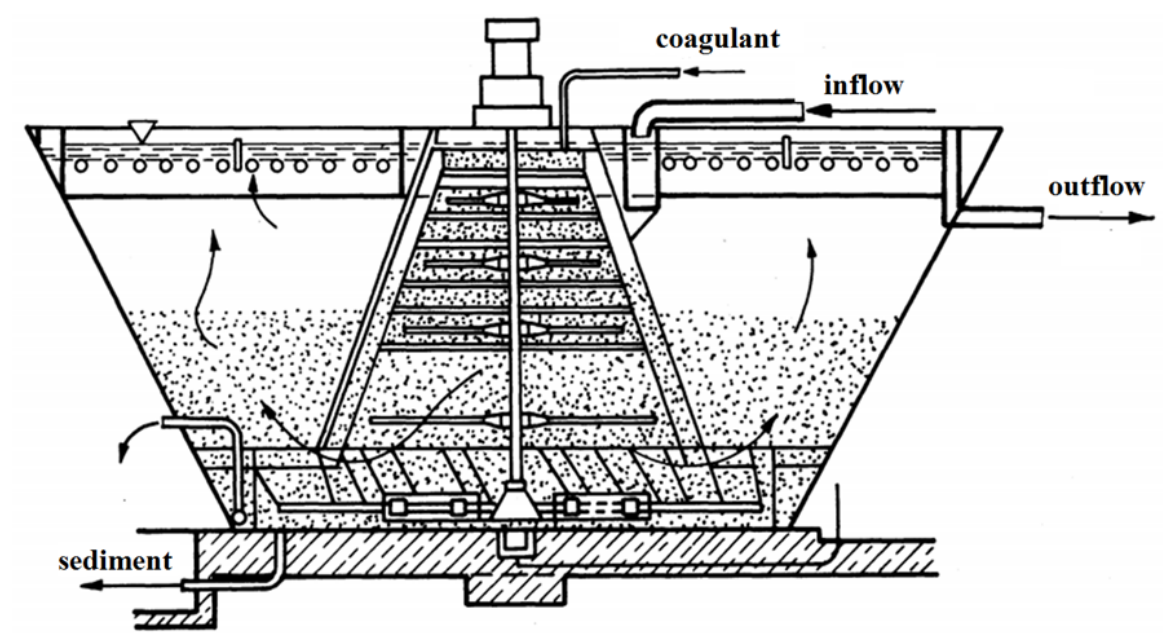

Figure 1: Reactor clarifier "Precipitator". 
(e.g. various types of water or wastewater) and the overall applied treatment scheme, coagulation-flocculation is usually included, either as pre-, or as post-treatment step [2], [5]. Properly carried coagulation not only provides a high degree of removal of colloidal suspensions and hard falling, as well as being associated with these other pollutants, including micropollutants. The effect of coagulation is an effective reduction of turbidity, colour intensity, indicating the organic impurities in that precursors of disinfection by-products and chemical oxidation and the content of a number of micropollutants, mainly present in the purified water in the form of sparingly soluble form or associates with the solid particles. The suspended particles vary considerably in source, composition charge, particle size, shape, and density. Correct application of coagulation and flocculation processes and selection of the coagulants depend upon understanding the interaction between these factors [1]. In practice, the coagulation is carried out by coagulants, where the hydrolysis products cause electrolyte coagulation and the coagulation by the colloid with opposite sign. Coagulation occur in two phases: in the first - after the addition of coagulant - run chemical and physical reactions leading to the destabilization of colloidal particles, and in the second phase, lasting much longer - flocculation - as a result of transport and collisions of destabilized particles, the flocs are created (Fig. 2). Flocs can be efficiently removed by the process of sedimentation/flotation and filtration [7]. After mixing process water is directed to clarifiers. Settling tanks are devices in which there is a process removing the suspension from purified water. They are used to easily retain the falling suspension, natural or produced by the coagulation process and chemical precipitation of sparingly soluble compounds. Contact settlers with suspended sediment are used to treatment the water by coagulation and are usually called clarifiers [8].

\section{MATHEMATICAL DESCRIPTION}

This paper presents the classification of flocs with the mathematical description of movement for particular fractions. It was carried out in order to determine the statistical drag coefficient value for groups of particles, which will allow for the modelling of a complex sediment suspension. The classification of flocs formed from the post-coagulation sediment was

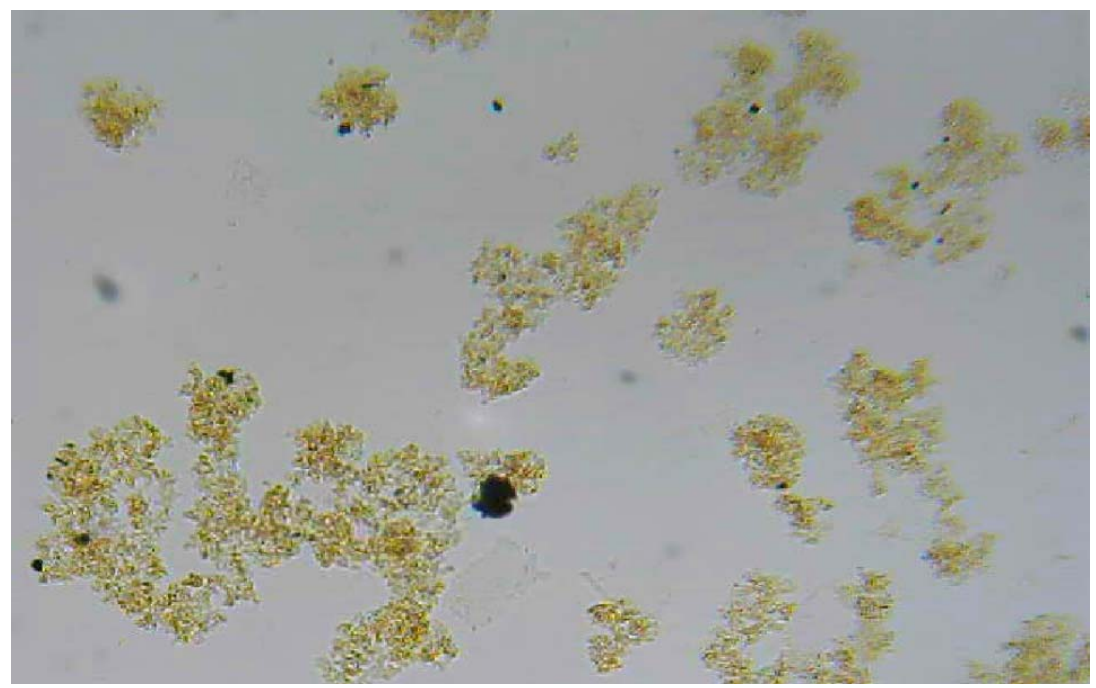

Figure 2: Post-coagulation suspension. 
carried out for the purposes of the research. Due to technological reasons, the settling time of the sediment is the most important parameter. Therefore, the classification proposed by the authors mainly focuses on the separation of groups of flocs according to their characteristic features affecting the settling velocity.

While examining the images, the authors decided to classify individual particles statistically. Flocs were divided into three characteristic groups. The classification takes into account the structure of a floc in terms of mass concentration (presence of macro-pores) and its shape. Flocs were divided into: compact particles, particles having a distinct mass concentration nucleus around which sediment crystallization networks are formed (flocks without mass concentration) and particles having a porous sponge structure (porous conglomerates).

The classification does not take into account all the characteristics. According to it, only the most important parameters of particles were considered. The occurrence and the size of pores in a single floc were assumed to be the criterion of division.

\subsection{Compact flocs}

Based on the flocs analysis, there were three separate types of particles characterized by a different settling way. Compact particles characterized a homogeneous structure. Their shape is similar to a sphere filled with the same microporous structure. Micro-pores are specific features of a post-coagulation material. Particles of this type fall in a straight line with a slight zigzag motion. Due to their small size and mass, their movement is repeatedly disrupted by fluid movement triggered by the movement of other particles, considerably higher in size, in their vicinity. Due to the fact that coagulation and sedimentation are important in the water treatment process, it is necessary to describe these processes by means of mathematical equations [8].

This description may take place only when the definition of basis forces acting on each particle is possible. Based on the presented division of particles into three types, the formation of different mathematical models for them is necessary. The speed of particles descent is defined as the balance of forces acting on a moving particle.

Therefore, the flocs presented in Fig. 3 are flocs with compact structure containing a large number of pores. These particles are characterized by the compact structure and relatively regular shape of a floc. Pores occurring in it are a natural feature of this type of suspensions. Settling of such particles can be described using the Stokes' law [7].

It is possible to formulate equations for compact particles that describe the descent process on the basis of Stokes equations. The basic distribution of forces acting on the descending
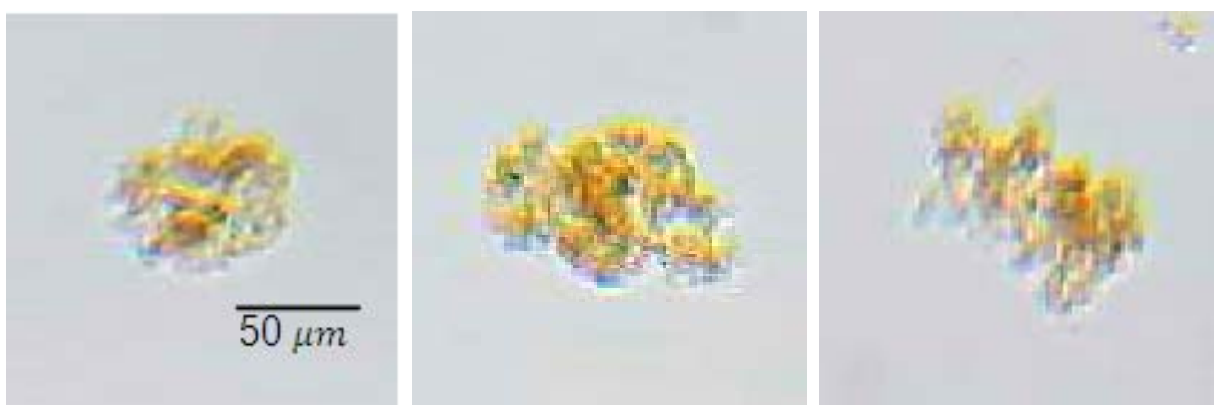

Figure 3: Compact structure of flocs. 


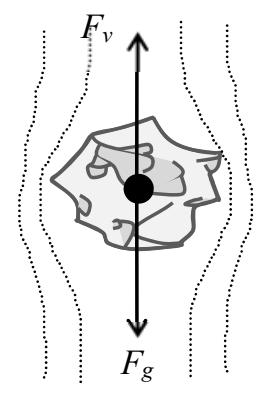

Figure 4: Forces acting on a compact particle.

particle is shown in Fig. 4. Two basic forces have an effect on the particle speed: gravity force always in the downward direction $\mathrm{F}_{\mathrm{g}}$ and drag force opposite to the direction of motion $\mathrm{F}_{\mathrm{v}}$.

Gravity force Fg, is defined according to Archimedes' principle (eqn (3)):

$$
F_{g}=\left(\rho_{s}-\rho_{1}\right) g V \text {, }
$$

where:

$\mathrm{g}$ - gravitational acceleration $[\mathrm{m} / \mathrm{s} 2]$,

$\mathrm{V}$ - particle volume [m3],

$\rho s, \rho 1-$ density of solid or liquid respectively $[\mathrm{kg} / \mathrm{m} 3]$.

Drag depends on the properties of the fluid and on the size, shape, and speed of the object. General formula for drag force $\mathrm{Fv}$ is shown in eqn (4):

$$
\mathrm{F}_{\mathrm{v}}=\mathrm{C}_{\mathrm{D}} \mathrm{A} \rho_{1} \frac{\mathrm{v}|\mathrm{v}|}{2}
$$

where:

$\mathrm{CD}$ - drag coefficient,

A - cross-sectional area in the horizontal plane [m2],

$\mathrm{v}-$ speed of descent $[\mathrm{m} / \mathrm{s}]$.

\subsection{Star-shaped particles}

The second group of particles (star-shaped particles) has a distinct mass concentration nucleus, around which sediment crystallization networks are formed (flocs without mass concentration) (Fig. 5). Protruding arms cause the particle to move in a circular motion around its own axis while falling. The circular motion causes the particle, whose direction is constantly changing, to be influenced by the Magnus effect. This effect causes the particles of this type to fall in a spiral motion. Over time, star-shaped particles connect to create bigger agglomerates. In case of big agglomerates, the circular motion around their own axis gradually disappears, and particles of this type fall in a straight line. They are characterised by considerably big macro-pores, through which fluid may flow during their motion. This type of structure is similar to fluid flow through a porous medium (Fig. 6).

Due to various shapes, it is necessary to determine an appropriate drag coefficient experimentally. The star-shaped particles have nucleus with concentrated mass surrounded by a number of sediment crystallization arms (Fig. 6). In case of such particles, the weight of a nucleus mainly affects the settling velocity. However, irregular crystallization arms change 
the direction in which particles settle. A non-linear trajectory significantly extends both settling path and time. The mathematical description of such particles requires much more complex mathematical models.

In case of star-shaped particles, the particle in motion rotates around its own axis. It causes lateral force to occur, which changes the direction of the particle descent. Due to the fact that the shape of the particle is irregular, the velocity at which the particle rotates around its own axis is not constant. It brings the change in the lateral force value, which, consequently, causes the particle to fall in an irregular motion, which is similar to a spiral in shape. Due to the influence of lateral force, the mathematical model should take into account not only lateral force, but also the fact that the sense of drag force is always opposite to the direction of the motion. Since the particle descent does not take place in a straight line, both the sense and value of drag force will change and the descent velocity will be influenced by the horizontal component of this force (Fig. 6). The shape of moving particles has a direct impact on their velocity of descending. The determination of the correct value of drag coefficient $C_{D}$ is difficult due to its complexity. In the literature, many empirical dependencies are applied that allow for the determination of the drag coefficient for non-spherical particles [10], [11], [12].

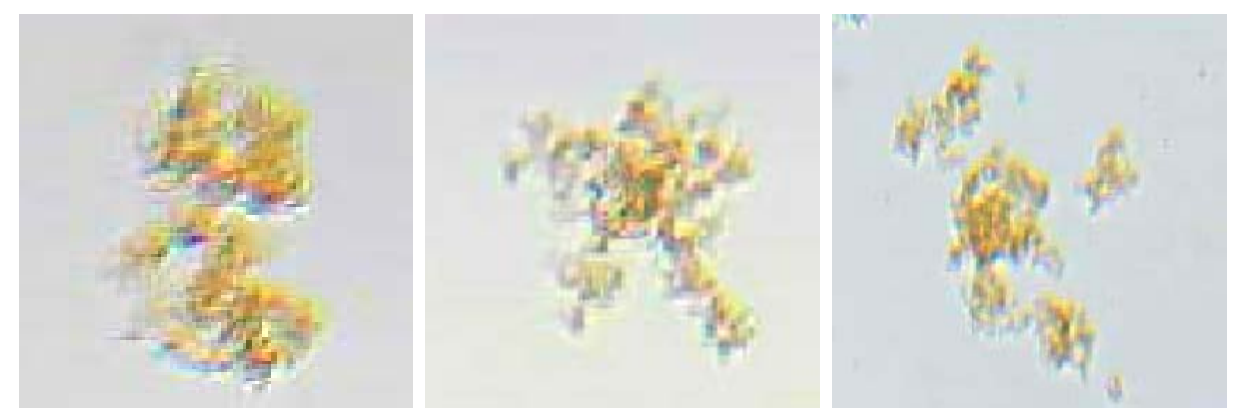

Figure 5: Flocks without mass concentration.

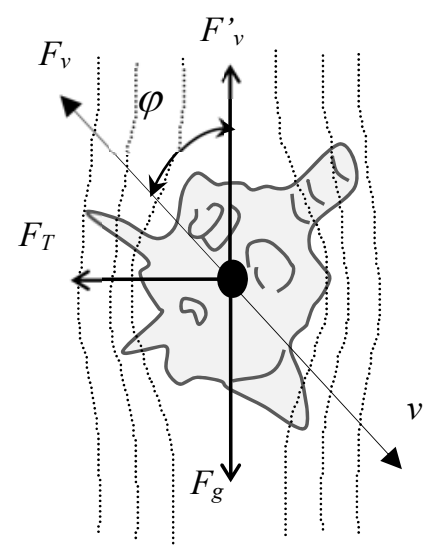

Figure 6: Forces acting on a star-shaped particle. 


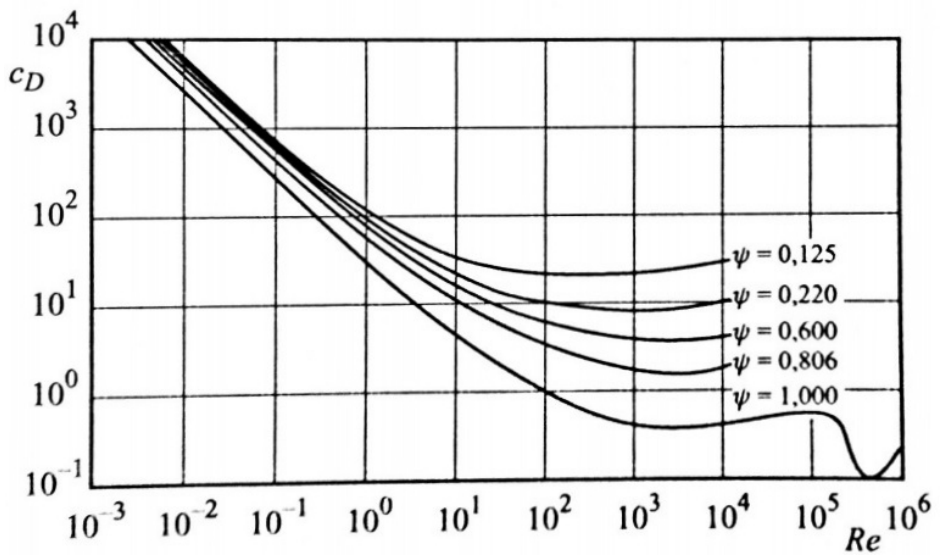

Figure 7: Dependence $C_{D}=f(\operatorname{Re}, \varphi)$ for non-spherical particles.

$$
F_{v}{ }^{\prime}=C_{D} A \rho_{l} \frac{v|v|}{2} \cos \varphi
$$

\subsection{Porous conglomerates}

The third group includes porous conglomerates consisting of the types of particles present in a given suspension, combined into clusters. Their structure resembles a sponge, they are very unstable and they can easily change their shape while settling. Fig. 8 presents the mass that is not concentrated in one place. The structure developed a porous structure (like a sponge). In addition, it was observed that the porous structure of flocs enables them to combine into larger conglomerates. According to the phenomenon of falling conglomerates, this type of structure is classified as a separate group in the classification of particles. The occurrence of a distinct porous structure will result in an additional flow of liquid through open pores.

Rotating around its own axis, the particle induces fluid movement, which induces lateral force $\mathrm{F}_{\mathrm{T}}$. The value of this force may be defined based on the Eötvös number. It describes a classic model of a stress-induced transverse rising force [12]:

$$
\mathrm{F}_{\mathrm{T}}=-\mathrm{C}_{\mathrm{T}} \rho_{\mathrm{C}}\left(\overline{\mathrm{v}_{\infty}}-\overline{\mathrm{v}_{\mathrm{C}}}\right) \times \operatorname{rot} \overline{\mathrm{v}_{\mathrm{C}}}
$$

Coefficient $\mathrm{C}_{\mathrm{T}}$ depends on the Reynolds number Re and a modified Eötvös number $\mathrm{Eo}_{\mathrm{d}}$, which is defined in eqn (7):

$$
\mathrm{Eo}_{\mathrm{d}}=\frac{\mathrm{g}\left(\rho_{\mathrm{s}}-\rho_{1}\right) \mathrm{d}_{\mathrm{M}}^{2}}{\sigma_{1}},
$$

where:

$\mathrm{d}_{\mathrm{M}}-$ maximum particle diameter in a horizontal section $[\mathrm{m}]$.

Agglomerates are formed when star-shaped flocs are connected. As a result of this process, cavities are created, which are called macro-pores. A network of macro-pores forms canals, through which liquid can flow during sedimentation. It allows for the stabilisation of the falling particles, which fall in a straight line. In this case, a similar model to the one for compact particles may be used. Gravity and drag force will have a dominant impact on the 


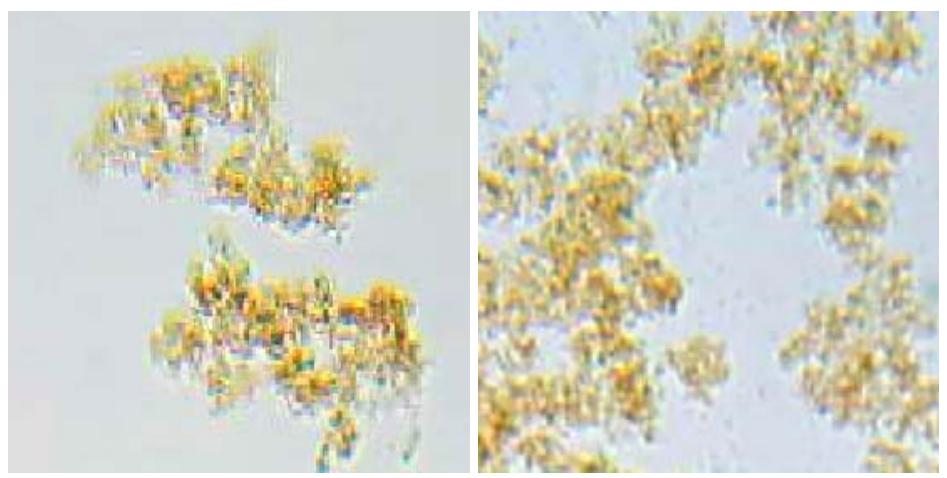

Figure 8: Porous conglomerates.

velocity of the descent. Since the liquid not only flows around the agglomerate but also through macro-pore canals, drag force should be defined in a different method.

Liquid flow through macro-pore canals is well described in models for a porous medium. Drag force for porous materials is described by the Darcy-Weisbach equation (eqn (8)) [10]:

$$
\mathrm{Q}=\frac{\Delta \mathrm{p}}{\mathrm{R}}
$$

where:

$\mathrm{R}$ - total drag of the porous baffle,

$\Delta \mathrm{p}$ - liquid pressure difference on both sides of the baffle.

Based on this formula, another formula for the porous material was derived, which is called the Leva equation (eqn (9)):

where:

$$
\Delta \mathrm{p}=\lambda \cdot \frac{\mathrm{h}}{\mathrm{d}_{\mathrm{z}}} \cdot \frac{(1-\varepsilon)^{3-\mathrm{n}}}{\varepsilon^{3}} \cdot \frac{\rho_{\mathrm{s}} \cdot \mathrm{w}^{2}}{2}
$$

$\lambda$ - drag coefficient

$\mathrm{w}$ - gas flow velocity through a porous material,

$\mathrm{d}_{\mathrm{z}}$ - equivalent diameter of the material,

$\varepsilon$ - porosity of the material.

The value of porosity and n-coefficient should be determined experimentally. To analyse the equations that describe the velocity of falling particles, one of the most basic parameters is density of the material, from which the particle was created. The main subject of this paper is to determine this parameter experimentally. The fact that post-coagulation sediment contains all types of particles poses a difficulty in the density determination of the material. Such a sediment contains a great number of macro-pores and there is fluid in the closed micro-pores.

\section{SUMMARY}

Sediment structures formed during coagulation process are diverse in terms of their shapes and degree of density. Taking the above into consideration, it can be observed that the modelling of this process is particularly difficult. There is a possibility of grouping flocs, taking their characteristics into account, and then forming general equations describing the movement of particles belonging to a given group. However, it is required to know the 
density of the material of which a floc is composed in order to properly describe the settling velocity of particles. This material has a closed microporous structure, which is a characteristic feature of the given sediment. In this work were consider only fractional model with no taking into consideration their interactions. This problem needs more studies. This issue is very important, especially because the settling velocities are low, so whirls cause minor interactions.

\section{REFERENCES}

[1] Burger, R., Hvistendahl Karlsen, K., Tory, E.M. \& Wendland, W.L., Model equations and instability regions for the sedimentation of polydisperse suspensions of spheres. Institute of Mathematics A, University of Stuttgart, p. 3, 2001.

[2] Basson, D.K., Berres, S. \& Bürger, R., On models of polydisperse sedimentation with particle-size-specific hendered-settling factors. Applied Mathematical Modelling, 33(4), p. 1815, 2009.

[3] Elgelhardt, L.T., Coagulation, Flocculation and Clarification of Drinking Water, Engelhardt, p. 5, 2010.

[4] Malakootian, M. \& Fatehizadeh, A., Color removal from water by coagulation/caustic soda and lime. Iran. J. Environ. Health. Sci. Eng., 7(3), p. 267, 2010.

[5] Ghernaout, D. \& Boucherit, A., Review of coagulation's rapid mixing for NOM removal. Journal of Research \& Developments in Chemistry, p. 2, 2015.

[6] Camacho, F.P., Bongiovani, M.C., Silva, M.O., Coldebella, P.F., Pessoa Amorim, M.T.S. \& Bergamasco, R., Coagulation/flocculation/flotation/nanofiltration processes using Moringa Oleifera as coagulant of eutrophized river. Chemical Engineering Transactions, 43, p. 1124, 2015.

[7] Xu, Y., Chen, T., Ciu, F. \& Shi, W., Effect of reused alum-humic-flocs on coagulation performance and floc characteristics formed by aluminum salt coagulants in humicacid water. Chemical Engineering Journal, 287, p. 226, 2016.

[8] Rząsa, R.M. \& Podgórni, E., Investigation of the effects of salinity and temperature on the removal of iron from water by aeration, filtration and coagulation. Pol. J. Environ. Stud., 23(6), p. 2157, 2014.

[9] Kowal, L.A. \& Świderska-Bróż, M., Water Treatment, PWN, p. 115, 1997.

[10] Chien, S.F., Settling velocity of irregularly shaped particles. SPE Drilling Completion, 9, p. 283, 1994.

[11] Ganser, G.H., A rational approach to drag prediction of spherical and non-spherical particles. Powder Technology, 77, p. 145, 1993.

[12] Haider, A. \& Levenspiel, O., Drag coefficient and terminal velocity of spherical and nonspherical particles. Powder Technology, 58, p. 67, 1989. 